\title{
Self-Dual and Decomposable Geometric Goppa Codes
}

\author{
Carlos Munuera and Ruud Pellikaan* \\ Appeared in: Eurocode 92, (P.Camion, P. Charpin and S. \\ Harari eds.), Udine, CISM Courses and Lectures 339, \\ Springer-Verlag, Wien-New York, 1993, 77-87.
}

\begin{abstract}
In this paper, a necessary and sufficient criterion for self-duality of geometric Goppa codes is given.

\section{Resumé}

On donne une condition necessaire et suffisante pour l'autodualité d'un code géométrique de Goppa.
\end{abstract}

\section{Introduction}

The aim of this note is to establish a necessary and sufficient criterion for self-duality of geometric Goppa codes. Self-dual codes play an important role in coding theory, so it is of interest to find conditions under which a code is self-dual.

*The first author is with the University of Valladolid, Department of Applied Mathematics (ETS de Arquitectura), Avda Salamanca S/N, 47014 Valladolid, Castilla (Spain), email cmunuera@cpd.uva.es and the last author is with the Eindhoven University of Technology, Department of Mathematics and Computing Science, P.O. Box 513, MB 5600 Eindhoven, The Netherlands, email ruudp@win.tue.nl 
Here we consider geometric Goppa codes, defined as follows:

Let $\mathcal{X}$ be a projective, nonsingular, absolutely irreducible curve of genus $g$ defined over the finite field $\mathbf{F}_{q}$. Let $\mathbf{F}_{q}(\mathcal{X})$ be the function field of $\mathcal{X}$ over $\mathbf{F}_{q}$ and $\Omega_{\mathcal{X}}$ the vector space of rational differential forms on $\mathcal{X}$ over $\mathbf{F}_{q}$.

Let $\mathcal{P}=\left\{P_{1}, \ldots, P_{n}\right\}$ be a set of $n$ distinct rational points on the curve $\mathcal{X}$. We fix the order of the $P_{i}$ and denote by $D$ the divisor $P_{1}+\ldots+P_{n}$. For a rational divisor $G$ on $\mathcal{X}$ of degree $m$ and support disjoint from $\mathcal{P}$ we consider the vector spaces $\mathcal{L}(G)=\left\{f \in \mathbf{F}_{q}(\mathcal{X})^{*} \mid(f) \geq-G\right\} \cup\{0\}$ and $\Omega(G)=\left\{\omega \in \Omega_{\mathcal{X}} \backslash\{0\} \mid(\omega) \geq G\right\} \cup\{0\}$. The algebraic-geometric or geometric Goppa codes associated to $\mathcal{P}$ and $G$ over $\mathbf{F}_{q}$ are defined by

$$
\begin{aligned}
C_{L}\left(\mathcal{X}, \mathcal{P}, G, \mathbf{F}_{q}\right) & =\left\{\left(f\left(P_{1}\right), \ldots, f\left(P_{n}\right)\right) \mid f \in L(G)\right\} \\
C_{\Omega}\left(\mathcal{X}, \mathcal{P}, G, \mathbf{F}_{q}\right) & =\left\{\left(\operatorname{res}_{P_{1}}(\omega), \ldots, \operatorname{res}_{P_{n}}(\omega)\right) \mid \omega \in \Omega(G-D)\right\},
\end{aligned}
$$

see Goppa [5], [6], [7] .

For the properties of geometric Goppa codes we refer to the textbook [11]. In order to be able to say something about the dimension and the minimum distance of these codes we restrict to the case $2 g-2<\operatorname{deg}(G)<n$, in which $C_{L}\left(\mathcal{X}, \mathcal{P}, G, \mathbf{F}_{q}\right)$ has dimension $\operatorname{deg}(G)+1-g$ and at least minimum distance $n-\operatorname{deg}(G)$; and $C_{\Omega}\left(\mathcal{X}, \mathcal{P}, G, \mathbf{F}_{q}\right)$ has dimension $n-\operatorname{deg}(G)-1+g$ and at least minimum distance $\operatorname{deg}(G)-2 g+2$.

In this paper we obtain a necessary and sufficient condition for self-duality of a geometric Goppa code $C_{L}\left(\mathcal{X}, \mathcal{P}, G, \mathbf{F}_{q}\right)$ in terms of $G$ and $D$. From now on we write, for short, $C(\mathcal{P}, G)$ instead of $C_{L}\left(\mathcal{X}, \mathcal{P}, G, \mathbf{F}_{q}\right)$ whenever it is clear which curve and field are meant.

Let us remember that a code $C(\mathcal{P}, G)$ is self-dual if it coincides with its dual $C(\mathcal{P}, G)^{\perp}$, that is with its orthogonal for the bilinear form

$$
<\mathbf{x}, \mathbf{y}>=\sum x_{i} y_{i}
$$

Let $\omega$ be a differential form on $\mathcal{X}$, and $W=(\omega)$. Since there is an isomorphism $\Omega(G-D) \cong \mathcal{L}(D+W-G)$, we have the following 
Proposition 1.1 There exists a differential form $\omega$ on $\mathcal{X}$ with simple poles and residue 1 at every $P_{i} \in \mathcal{P}$ such that $C(\mathcal{P}, G)^{\perp}=C(\mathcal{P}, D+W-G)$, where $W$ is the divisor of $\omega$.

A sufficient condition for self-duality follows from Proposition 1.1

Theorem 1.2 If there exists a differential form $\eta$ with simple poles and residue 1 at every $P_{i} \in \mathcal{P}$ such that $2 G=D+K$, where $K=(\eta)$, then $C(\mathcal{P}, G)$ is self dual.

Proof: Let $\omega$ be the differential form cited in 1.1. There exists a rational function $f$ such that $\eta=f \omega$; since both $\eta$ and $\omega$ have simple poles at every $P_{i}$ it is $v_{p_{i}}(f)=0$ and $f\left(P_{i}\right)=\operatorname{res}_{P_{i}}(\eta) / \operatorname{res}_{P_{i}}(\omega)=\operatorname{res}_{P_{i}}(\eta)=1$. Then $K=W+(f)$, so $D+W-G \sim D+K-G$ and $C(\mathcal{P}, G)=C(\mathcal{P}, D+K-G)=$ $C(\mathcal{P}, D+W-G)=C(\mathcal{P}, G)^{\perp}$; thus $C(\mathcal{P}, G)$ is self dual.

This result is due to H. Stichtenoth (see [9]). In some cases the condition stated there is also necessary. For example, in the $g>1$ case, Y. Driencourt and Stichtenoth (see [3] ) gave the following

Theorem 1.3 Let $g>1$. Suppose $G$ is a divisor with:

a) $G \geq 0$;

b) $\operatorname{deg}(G) \geq 4 g-1$; and

c) $v_{P}(G) \geq \operatorname{deg}(G)-2 g+1$ for all places $P \leq G$;

then, the converse of 1.2 holds.

Later this result has been generalized by C.P. Xing (see [12] ) by assuming $n>6 g-4$ only.

However the converse of 1.2 is not always true. For instance, in the same paper [3] a counterexample with $g=1$ and $n=4=2 g+2$ is found. Moreover, as shown in [8], it is possible to get a similar example over $\mathbf{F}_{q}$, where $q$ is an even power of 2 , with a code arising from an hyperelliptic curve of genus $g=\frac{1}{2} q-1$, and of length $n=2 g+2$.

Furthermore, the converse of Theorem 1.2 is not true for so-called decomposable codes, which we study in the next section. In section 3 we prove the converse of (1.2). Both sections, 2 and 3, are based on the paper [8] by C. Munuera and R. Pellikaan. Self-dual Goppa codes have been studied in [1], [2], [3] and [9] too. 


\section{Decomposable Codes}

Definition 2.1 If $C_{1}$ is an $\left[n_{1}, k_{1}\right]$ code, and $C_{2}$ is an $\left[n_{2}, k_{2}\right]$ code, then we say that $C$ is the direct sum of $C_{1}$ and $C_{2}$ if (up to reordering of coordinates)

$$
C=\left\{(\mathbf{x}, \mathbf{y}) \mid \mathbf{x} \in C_{1}, \mathbf{y} \in C_{2}\right\} .
$$

We denote this by $C=C_{1} \oplus C_{2}$. If moreover $C_{1}$ and $C_{2}$ are nonzero, then we say that $C$ decomposes into $C_{1}$ and $C_{2}$. We call a linear code $C$ decomposable if there exist nonzero codes $C_{1}$ and $C_{2}$ such that $C$ decomposes into $C_{1}$ and $C_{2}$.

Remark 2.2 1) The code $C$ decomposes into $C_{1}$ and $C_{2}$ if and only if (up to reordering of coordinates) $C$ has a generator matrix of the form

$$
M=\left(\begin{array}{cc}
M_{1} & 0 \\
0 & M_{2}
\end{array}\right),
$$

where $M_{1}$ and $M_{2}$ are nonempty generator matrices for $C_{1}$ and $C_{2}$, respectively.

2) If $C$ decomposes into an $\left[n_{1}, k_{1}, d_{1}\right]$ and an $\left[n_{2}, k_{2}, d_{2}\right]$ code, then obviously $C$ is an $\left[n_{1}+n_{2}, k_{1}+k_{2}, d\right]$ code, where $d=\min \left(d_{1}, d_{2}\right)$. Hence

$$
d \leq \frac{n-k}{2}+1
$$

by the Singleton bound. Thus there are no MDS decomposable codes.

Example 2.3 Let $C$ be a code of minimum distance one and length greater than one. If $\mathbf{x}$ is a codeword of weight one and $\left\{\mathbf{x}, \mathbf{y}_{1}, \ldots, \mathbf{y}_{k-1}\right\}$ is a basis of $C$, then so is $\left\{\mathbf{x}, \mathbf{y}_{1}-\lambda_{1} \mathbf{x}_{1}, \ldots, \mathbf{y}_{k-1}-\lambda_{k-1} \mathbf{x}_{1}\right\}$. Thus $C$ has a generator matrix of the form

$$
\left(\begin{array}{cc}
1 & 0 \\
0 & M^{\prime}
\end{array}\right)
$$

where $M^{\prime}$ is an $(n-1) \times(k-1)$ matrix, so $C$ is decomposable. We say that codes of minimum distance one and length greater than one are trivial decomposable.

In the following we will discuss nontrivial decomposable geometric Goppa codes. 
Proposition 2.4 Let $G$ be a divisor such that $\operatorname{deg}(G)<n$. Then the following statements are equivalent:

1) $C(\mathcal{P}, G)$ is a decomposable code.

2) There are two nonzero effective divisors $D_{1}$ and $D_{2}$ such that $D_{1}+D_{2}=D$, both $\mathcal{L}\left(G-D_{1}\right)$ and $\mathcal{L}\left(G-D_{2}\right)$ are not zero, and $\mathcal{L}(G)=\mathcal{L}\left(G-D_{1}\right) \oplus \mathcal{L}(G-$ $\left.D_{2}\right)$.

Proof: We have $\mathcal{L}\left(G-D_{1}\right) \cap \mathcal{L}\left(G-D_{2}\right)=\mathcal{L}(G-D)=(0)$, since $\operatorname{deg}(G)<n$. Both $\mathcal{L}\left(G-D_{1}\right)$ and $\mathcal{L}\left(G-D_{2}\right)$ are subspaces of $\mathcal{L}(G)$. If $C$ is decomposable, then there exist two effective divisors $D_{1}$ and $D_{2}$ such that $D_{1}+D_{2}=D$, and a basis $\left\{f_{1}, \ldots, f_{s}, g_{1}, \ldots, g_{t}\right\}$ with $\left(f_{i}\right) \geq D_{2}$ for $i=1, \ldots, s$ and $\left(g_{i}\right) \geq D_{1}$ for $i=1, \ldots, t$, so $\left\{f_{1}, \ldots, f_{s}\right\} \subseteq \mathcal{L}\left(G-D_{2}\right)$ and $\left\{g_{1}, \ldots, g_{t}\right\} \subseteq \mathcal{L}\left(G-D_{1}\right)$ and (2) is proved. Conversely, if $\mathcal{L}\left(G-D_{1}\right)=<g_{1}, \ldots, g_{s}>$ and $\mathcal{L}\left(G-D_{2}\right)=$ $<f_{1}, \ldots, f_{t}>$, then $\mathcal{L}(G)=<f_{1}, \ldots, f_{s}, g_{1}, \ldots, g_{t}>$ so $C(\mathcal{P}, G)$ decomposes into $C\left(\operatorname{supp}\left(D_{1}\right), G-D_{2}\right)$ and $C\left(\operatorname{supp}\left(D_{2}\right), G-D_{1}\right)$.

Corollary 2.5 If $\operatorname{deg}(G)<n$ and $C(\mathcal{P}, G)$ is a decomposable code of dimension $k$, then $k \leq \ell(2 G-D)+1$.

Proof: For two effective divisors $E_{1}, E_{2}$ we have $\ell\left(E_{1}\right)+l\left(E_{2}\right) \leq \ell\left(E_{1}+\right.$ $\left.E_{2}\right)+1$, see [11]. According to Proposition 2.4 there are two effective divisors $D_{1}$ and $D_{2}$ such that $D=D_{1}+D_{2}$ and $l\left(G-D_{1}\right)>0$ and $\ell\left(G-D_{2}\right)>0$, so

$$
k=\ell\left(G-D_{1}\right)+\ell\left(G-D_{2}\right) \leq \ell(2 G-D)+1 .
$$

Corollary 2.6 If $\operatorname{deg}(G)<n$ and $C(\mathcal{P}, G)$ is nontrivial decomposable, then $n \leq 2 g+2$.

Proof: Let $m=\operatorname{deg}(G)$. Suppose $C(\mathcal{P}, G)$ decomposes into $C\left(\operatorname{supp}\left(D_{1}\right), G\right)$ and $C\left(\operatorname{supp}\left(D_{2}\right), G\right)$, with $n_{1}=\operatorname{deg}\left(D_{1}\right)$ and $n_{2}=\operatorname{deg}\left(D_{2}\right)$. We may assume that $n_{1} \leq n_{2}$. It is not possible that both $m-n_{1}<0$ and $m-n_{2}<0$, otherwise $\ell\left(G-D_{i}\right)=0$ for $i=1,2$, so $\ell(G)=0$, by Proposition 2.4. But the code is not zero. Now there are several cases.

Case 1a: If $m-n_{1}>2 g-2$ and $m-n_{2}<0$, then $m+1-g \leq m-n_{1}+1-g$, by Proposition 2.4 and the Riemann-Roch Theorem. Hence $n_{1}=1$. Thus the code is trivial decomposable, which is a contradiction. 
Case 1b: If $m-n_{1}>2 g-2$ and $0 \leq m-n_{2} \leq 2 g-2$, then

$$
m+1-g \leq m-n_{1}+1-g+\frac{m-n_{2}}{2}+1,
$$

by Proposition 2.4, the Riemann-Roch theorem and Clifford's theorem. So $n+n_{1} \leq m+2$. Moreover $\operatorname{deg}(G)<n$, hence $m=n-1$ and $n_{1}=1$. Thus the code is trivial decomposable, which is a contradiction.

Case 2: If $m-n_{2}>2 g-2$ then

$$
m+1-g=m-n_{1}+1-g+m-n_{2}+1-g,
$$

so $n+g=m+1$. Moreover $\operatorname{deg}(G)<n$, hence $m=n-1$ and $g=0$. Thus the code is MDS and decomposable, so it is trivial decomposable, which is a contradiction.

Case 3a: If $0 \leq m-n_{1} \leq 2 g-2$ and $m-n_{2}<0$.

Case 3b: If $m-n_{1} \leq 2 g-2$ and $0 \leq m-n_{2}$, then $0 \leq m-n_{i} \leq 2 g-2$ for $i=1,2$, since $n_{1} \leq n_{2}$ and either $0 \leq m-n_{1}$ or $0 \leq m-n_{2}$. Thus

$$
m+1-g \leq \frac{m-n_{1}}{2}+1+\frac{m-n_{2}}{2}+1,
$$

so $n \leq 2 g+2$.

Example 2.7 Let $G$ be a divisor of degree $m$ on a curve $\mathcal{X}$ of genus $g$. Suppose $m<n=2 g+2$. If

1) $G$ has degree $2 g$ and there are two nonzero effective divisors $D_{1}$ and $D_{2}$ such that $D_{1}+D_{2}=D$ and $G-D_{1}$ is canonical and $G-D_{2}$ is principal, or 2) $G$ is not special and $\mathcal{X}$ is hyperelliptic and there are two nonzero effective divisors $D_{1}$ and $D_{2}$ such that $D_{1}+D_{2}=D$ and both $G-D_{1}$ and $G-D_{2}$ are hyperelliptic divisors,

then $C(D, G)$ is decomposable.

This is a direct consequence of Proposition 2.4.

Conversely, if $C(\mathcal{P}, G)$ is a decomposable code of length $n=2 g+2$ and dimension $k=g+1$, it is $\operatorname{deg}(G)=2 g$, so according to 2.5 it is

$$
\begin{aligned}
\operatorname{deg}(2 G-D) & =2 g-2 \\
\ell(2 G-D) & =g
\end{aligned}
$$


so $2 G-D$ is a canonical divisor with simple poles at every point in $\mathcal{P}$, $2 G-D=(\omega)$. If $C(\mathcal{P}, G)$ decomposes about the codes made from the points in $\mathcal{P}_{1}, \mathcal{P}_{2}\left(\mathcal{P}_{1} \cap \mathcal{P}_{2}=\emptyset, \mathcal{P}_{1} \cup \mathcal{P}_{2}=\mathcal{P}\right)$, in such a way that the residues of $\omega$ are equal to $\lambda_{1}$ at every point in $\mathcal{P}_{1}$, and the residues of $\omega$ are equal to $\lambda_{2}$ at every point in $\mathcal{P}_{2}$, with $\lambda_{1} \neq \lambda_{2}$, then according to 1.2 , the code is self-dual but the converse of 1.2 fails.

In the same way the converse of 1.2 is not true, in general, for self-dual decomposable geometric Goppa codes.

\section{Equality of codes and equivalence of divi- sors}

In order to prove the converse of 1.2 and according to the results obtained in the last section, we must restrict ourselves to $n>2 g+2$ (and $\operatorname{deg}(G)<n$ ), in which case there are no decomposable nontrivial geometric Goppa codes.

The problem we want to solve, can be viewed as a particular case of the following: given two divisors $G, H$ on $\mathcal{X}$ with support disjoint from $\mathcal{P}$, and such that $C(\mathcal{P}, G)=C(\mathcal{P}, H)$, then, which relationship between $G$ and $H$ is there?

This problem has been treated by C.P. Xing in [12]. In his work he assumes $2 g-1<\operatorname{deg}(G)=\operatorname{deg}(H)<n-1$ and $n>2 g+2$. In this case he obtains

Proposition 3.1 Suppose $n>2 g+2$. Let $G$ and $H$ be two effective divisors with support disjoint from $\mathcal{P}$ on the curve $\mathcal{X}$. If $2 g-1<\operatorname{deg}(G), \operatorname{deg}(H)<$ $n-1$, then $C(\mathcal{P}, G)=C(\mathcal{P}, H)$ if and only if $G=H$.

However equality is not the most general situation in which two divisors provide the same code as we shall see. Firstly we define a special case of linear equivalence of divisors

Definition 3.2 1) Two divisors $G, H$ on $\mathcal{X}$ are called equivalent about $\mathcal{P}$, denoted $G \sim_{\mathcal{P}} H$, if there exists a rational function $f$ such that $f\left(P_{i}\right)=1$ 
for every $P_{i} \in \mathcal{P}$ and $H-G=(f)$.

2) Let $C$ be a linear code in $\mathbf{F}_{q}^{n}$ and $\lambda=\left(\lambda_{1}, \ldots, \lambda_{n}\right)$ an $n$-tuple of nonzero elements in $\mathbf{F}_{q}$. For every $\mathbf{x}=\left(x_{1}, \ldots, x_{n}\right) \in C$, define $\lambda \mathbf{x}=\left(\lambda_{1} x_{1}, \ldots, \lambda_{n} x_{n}\right)$ and $\lambda C=\{\lambda \mathbf{x} \mid \mathbf{x} \in C\}$.

Remark 3.3 We can view $\lambda$ as a linear map in $\mathbf{F}_{q}^{n}$ leaving the Hamming metric invariant. Note that a linear map of $\mathbf{F}_{q}^{n}$ leaves the Hamming metric invariant if and only if it is of the form $\lambda \sigma$ for some permutation $\sigma$ of $\{1, \ldots, n\}$.

The relation between the notion of equivalence about $\mathcal{P}$ and our problem is given by the following property (see [10])

Proposition 3.4 Let $G, H$ be two divisors on $\mathcal{X}$ with support disjoint from $\mathcal{P}$. If $G \sim_{\mathcal{P}} H$ then $C(\mathcal{P}, G)=C(\mathcal{P}, H)$.

Proof: If $H-G=(f)$ then the map

$$
\mathcal{L}(H) \longrightarrow \mathcal{L}(G) ; h \mapsto f h
$$

is an isomorphism of vector spaces. Thus $\mathcal{L}(G)=\{f h \mid h \in \mathcal{L}(H)\}$, so $C(\mathcal{P}, G)=\left(f\left(P_{1}\right), \ldots, f\left(P_{n}\right)\right) C(\mathcal{P}, H)=(1, \ldots, 1) C(\mathcal{P}, H)=C(\mathcal{P}, H)$

In the rest of this section we shall prove the converse of 3.4 .

Proposition 3.5 Assume $n>2 g+2$ and $2 g-1<\operatorname{deg}(G)<n-1$. If there is a word in $C(\mathcal{P}, G)$ with weight $n$ then $C(\mathcal{P}, G)=C(\mathcal{P}, H)$ if and only if $G \sim_{\mathcal{P}} H$

Proof: After 3.4 we have to prove only one direction of the assertion. Let $\left(x_{1}, \ldots, x_{n}\right) \in C(\mathcal{P}, H)$ be a codeword of weight $n$ and let $f \in \mathcal{L}(G), h \in \mathcal{L}(H)$ be the functions such that $f\left(P_{i}\right)=x_{i}=h\left(P_{i}\right)$ for every $P_{i} \in \mathcal{P}$. Let us consider the divisors $G^{\prime}=G+(f), H^{\prime}=H+(h)$. It is clear that $G^{\prime}$ and $H^{\prime}$ are effective divisors with support disjoint with $\mathcal{P}$. Furthermore, as we have seen in 3.4 it is

$$
C(\mathcal{P}, G)=\left(f\left(P_{1}\right), \ldots, f\left(P_{n}\right)\right) C\left(\mathcal{P}, G^{\prime}\right)=\left(x_{1}, \ldots, x_{n}\right) C\left(\mathcal{P}, G^{\prime}\right)
$$

and

$$
C(\mathcal{P}, H)=\left(h\left(P_{1}\right), \ldots, h\left(P_{n}\right)\right) C\left(\mathcal{P}, H^{\prime}\right)=\left(x_{1}, \ldots, x_{n}\right) C\left(\mathcal{P}, H^{\prime}\right)
$$


so

$$
\left(x_{1}, \ldots, x_{n}\right) C\left(\mathcal{P}, G^{\prime}\right)=\left(x_{1}, \ldots, x_{n}\right) C\left(\mathcal{P}, H^{\prime}\right)
$$

that is $C\left(\mathcal{P}, G^{\prime}\right)=C\left(\mathcal{P}, H^{\prime}\right)$. Now, according to 3.1 , it is $G^{\prime}=H^{\prime}$, so $G-H=(h / f)$, and $G \sim_{\mathcal{P}} H$

Remark 3.6 The above proposition is not true without the restriction on the degree of the divisors $2 g-1<\operatorname{deg}(G)<n-1$ as we can see as follows: let $K$ be a canonical divisor and $P, Q$ two rational points not in $\mathcal{P}$. Since $\ell(K+P)=\ell(K+Q)=\ell(K)$ it is $\mathcal{L}(K+P)=\mathcal{L}(K+Q)$ so $C(\mathcal{P}, K+P)=C(\mathcal{P}, K+Q)$ but $K+P \nsim K+Q$.

The result stated in 3.5 seems to depend on the existence of codewords with weight large enough but, in fact, this condition is superfluous.

Proposition 3.7 If $C$ is a code in $\mathbf{F}_{q}^{n}$ not contained in a coordinate hyperplane, and $n<q$, then there is a codeword in $C$ with weight $n$.

Proof: For every $i=1, \ldots, n$ let $C_{i}=C \cap\left(x_{i}=0\right)$. Since $C$ is not contained in any hyperplane of the form $x_{i}=0$, it is $C_{i} \neq C$ for all $i$. If there is not a codeword with weight $n$ then $C \subseteq \cup_{i=1}^{n} C_{i}$, so, by taking cardinalities we have $q^{k} \leq n q^{k-1}$ (where $k=\operatorname{dim} C$ ), that is $q \leq n$

Corollary 3.8 If $2 g-1<\operatorname{deg}(G)<n-1$ and $n<q$, then there exists in $C(\mathcal{P}, G)$ a codeword with weight $n$.

Proof: If $C(\mathcal{P}, G)$ is contained in a hyperplane $x_{i}=0$, then $P_{i}$ is a base point for $G$, but since $2 g-1<\operatorname{deg}(G), G$ is base point free

For given a positive integer $r$ let us consider the vector space over $\mathbf{F}_{q^{r}}$

$$
\mathcal{L}\left(G, \mathbf{F}_{q^{r}}\right)=\left\{f \in \mathbf{F}_{q^{r}}(\mathcal{X})^{*} \mid(f) \geq-G\right\} \cup\{0\}
$$

and the code $C\left(\mathcal{X}, \mathcal{P}, G, \mathbf{F}_{q^{r}}\right)$, image of the map

$$
\mathcal{L}\left(G, \mathbf{F}_{q^{r}}\right) \longrightarrow \mathbf{F}_{q^{r}}^{n} ; f \mapsto\left(f\left(P_{1}\right), \ldots, f\left(P_{n}\right)\right) .
$$

Lemma 3.9 For every divisor $G$ rational over $\mathbf{F}_{q}$, it is $\operatorname{dim}_{\mathbf{F}_{q}}\left(\mathcal{L}\left(G, \mathbf{F}_{q}\right)\right)=$ $\operatorname{dim}_{\mathbf{F}_{q^{r}}}\left(\mathcal{L}\left(G, \mathbf{F}_{q^{r}}\right)\right)$. 
Proof: See [11], chap. (2.31)

Lemma 3.10 Let $G, H$ be two divisors on $\mathcal{X}$, rational over $\mathbf{F}_{q}$. If $C\left(\mathcal{X}, \mathcal{P}, G, \mathbf{F}_{q}\right)=$ $C\left(\mathcal{X}, \mathcal{P}, G, \mathbf{F}_{q}\right)$ then $C\left(\mathcal{X}, \mathcal{P}, G, \mathbf{F}_{q^{r}}\right)=C\left(\mathcal{X}, \mathcal{P}, G, \mathbf{F}_{q^{r}}\right)$.

Proof: Let $\left\{f_{1}, \ldots, f_{k}\right\}$ be a basis for $\mathcal{L}\left(G, \mathbf{F}_{q}\right)$. Since $\mathcal{L}\left(G, \mathbf{F}_{q}\right) \subseteq \mathcal{L}\left(G, \mathbf{F}_{q^{r}}\right)$, according to 3.9 , it is a basis for $\mathcal{L}\left(G, \mathbf{F}_{q^{r}}\right)$ too, so both, $C\left(\mathcal{X}, \mathcal{P}, G, \mathbf{F}_{q}\right)$ and $C\left(\mathcal{X}, \mathcal{P}, G, \mathbf{F}_{q^{r}}\right)$ have the same generator matrix

At this moment we are able to prove the following

Theorem 3.11 Assume $n>2 g+2$. If $2 g-1<\operatorname{deg}(G)$, $\operatorname{deg}(H)<n-1$ then $C(\mathcal{P}, G)=C(\mathcal{P}, H)$ if and only if $G \sim_{\mathcal{P}} H$.

Proof: After 3.4 it is enough to prove the 'only if' part. Assume $C(\mathcal{P}, G)=$ $C(\mathcal{P}, H)$ and take an integer $r$ such that $q^{r}>n$. According to 3.7 there is a codeword of weight $n$ in $C\left(\mathcal{X}, \mathcal{P}, G, \mathbf{F}_{q^{r}}\right)$, so from 3.5 it is $G \sim_{\mathcal{P}} H$ over $\mathbf{F}_{q^{r}}$. Thus $\ell\left(G-H, \mathbf{F}_{q^{r}}\right)=1$, and there is a function $f \in \mathcal{L}\left(G-H, \mathbf{F}_{q^{r}}\right)$ such that $f\left(P_{1}\right)=\ldots=f\left(P_{n}\right)=1$. Since $G$ and $H$ are rational over $\mathbf{F}_{q}$ it is $\ell\left(G-H, \mathbf{F}_{q}\right)=1$ according to 3.9 , so there exists a nonzero function $f^{\prime} \in \mathcal{L}\left(G-H, \mathbf{F}_{q}\right)$, so $f=\lambda f^{\prime}$ for some $\lambda \in \mathbf{F}_{q^{r}}^{*}$, so $f^{\prime}\left(P_{1}\right)=1 / \lambda$, and (after scaling $f^{\prime}$ if necessary) we can assume $f^{\prime}\left(P_{1}\right)=1$, so $\lambda=1$, so $f=f^{\prime}$, and finally $G \sim_{\mathcal{P}} H$ over $\mathbf{F}_{q}$.

Let us return now to our problem of settling the converse of 1.2 for the self-duality of geometric Goppa codes.

As we know, the dimension of a code $C(\mathcal{P}, G)$ is $k=\ell(G)-\ell(G-D)$, thus,

a) if $\operatorname{deg}(G) \geq n$ then $k \geq n-g$;

b) if $2 g-2<\operatorname{deg}(G)<n$ then $g \geq k \geq n-g$; and

c) if $\operatorname{deg}(G) \leq 2 g-2$ then $k \leq g$;

(these results follow from Riemann-Roch and Clifford's theorems) so then, taking into account that $k=\frac{n}{2}$ when $C(\mathcal{P}, G)$ is self dual, it is $k=\frac{n}{2}$ $2 g-2<\operatorname{deg}(G)<n$, and then $\frac{n}{2}=k=l(G)=\operatorname{deg}(G)+1-g$. Since we are assuming $n>2 g+2$, in fact it is $2 g-1<\operatorname{deg}(G)<n-1$ and we can apply theorem 3.11 (note that when $n>2 g+2$ every self-dual geometric Goppa code has minimum distance $d>1$, so it is not trivial decomposable). Thus we obtain the following 
Theorem 3.12 Assume $n>2 g+2$. The code $C(\mathcal{P}, G)$ is self dual if and only if there exists a differential form $\eta$ with simple poles and residue 1 at every $P_{i} \in \mathcal{P}$ such that $2 G=D+K$, where $K=(\eta)$.

Proof: Since $C^{\perp}(\mathcal{P}, G)=C(\mathcal{P}, D+W-G)$, where $W=(\omega)$, $\omega$ with simple poles and residue 1 at every $P_{i} \in \mathcal{P}$, according to 3.11 there is a rational function $f$ such that $f\left(P_{i}\right)=1$ for all $P_{i} \in \mathcal{P}$ and $2 G=D+W+(f)$. Then it is enough to take $\eta=f \omega$

When $n \leq 2 g+2$ the conclusion of the theorem fails as said in sections 1 and 2 .

\section{References}

[1] Y. Driencourt and J.F. Michon, Rapport sur les codes géométriques, oct. 1986.

[2] Y. Driencourt and J.F. Michon, Remarques sur les codes géométriques, C.R. Acad. Sc. Paris 301 (1985), 15-17.

[3] Y. Driencourt and H. Stichtenoth, A criterion for self-duality of geometric codes, Communications in Algebra 17(4) (1989), 885-898.

[4] V.D. Goppa, Codes associated with divisors, Probl. Peredachi Inform. 13(1) (1977), 33-39. Translation: Probl. Inform. Transmission 13 (1977), $22-26$.

[5] V.D. Goppa, Codes on algebraic curves, Dokl. Akad. Nauk SSSR 259 (1981), 1289-1290. Translation: Soviet Math. Dokl. 24 (1981), 170-172.

[6] V.D. Goppa, Algebraico-geometric codes, Izv. Akad. Nauk SSSR 46 (1982), Translation: Math. USSR Izvestija 21 (1983), 75-91.

[7] V.D. Goppa, Codes and information, Russian Math. Surveys 39 (1984), 87-141.

[8] C. Munuera and R. Pellikaan, Equality of geometric Goppa codes and equivalence of divisors, preprint, 1992. 
[9] H. Stichtenoth, Self-dual Goppa codes, J. Pure Appl. Algebra 55 (1988), 199-211.

[10] H. Stichtenoth, The automorphisms of geometric Goppa codes, J. Algebra 130 (1990), 113-121.

[11] M. Tsfasman and S. Vladut, Algebraic Geometric Codes, Kluwer Ac. Publ, Dordrecht, 1991.

[12] C.-P. Xing, When are two geometric Goppa codes equal ?, IEEE Trans. Information Theory IT-38 (1992), 1140-1142. 